CACHEXIA

\section{A bit of muscle}

Almost one-third of cancer deaths are due to cachexia — a wasting condition characterized by loss of skeletal muscle — and not due to tumour burden. Although we know some key cachetic factors — such as the cytokines tumour necrosis factor- $\alpha$ (TNF $\alpha)$, interferon- $\gamma$ (IFN $\gamma)$ and interleukin-6 (IL6) - their targets within muscle have not been identified. Denis Guttridge and colleagues now report that cachetic factors selectively target the myosin heavy chain (MYHC), and find that the mechanism by which they reduce myosin expression is factor-dependent.

The authors studied the effect of TNF $\alpha$ in combination with IFN $\gamma$ on the expression of myofibrillar proteins in a mouse myotube culture model and in primary mouse myotubes. Only expression of MYHC - which accounts for about $40 \%$ of myofibrillar protein in muscle — was decreased. Although TNF $\alpha$ or IFN $\gamma$ alone had little effect, together they reduced mRNA levels of both Myhc and the myocyte regulatory factor Myod, which controls transcriptional activation of a key isoform of MYHC. Exogenous expression of MYOD rescued $M y h c$ transcription in the presence of TNF $\alpha$ and IFN $\gamma$, indicating that the cytokines act by inhibiting MYOD synthesis. MYHC protein turnover was not altered in the myotube models. Analysis of the effects of TNF $\alpha$ plus IFN $\gamma$ in vivo, using cytokine-expressing and control chinese hamster ovary cells injected intramuscularly into mice, confirmed the selective decrease in Myhc mRNA.

So, does this mechanism of reduction of muscle protein hold true in the context of cancer? To assess this, Guttridge and colleagues examined MYHC in an established cancer cachexia model - the colon-26 adenocarcinoma mouse. Although this model is dependent on IL6 rather than TNF $\alpha$ or IFN $\gamma$, the authors reasoned that MYHC would be a selective target no matter which factor induced the cachexia, and they did indeed show selective reduction of MYHC. However, interestingly, Myhc mRNA levels were not reduced in the cachetic muscles. Instead, they found that reduction occurred at the protein level. Expression of the degradation-targeting protein ubiquitin, together with muscle-specific ubiquitin-pathway genes, was increased in cachetic muscles. In addition, immunoprecipitation studies showed that MYHC fragments were present in the cachetic muscles, and in some muscles less myosin was co-precipitated with ubiquitin, indicating that the myosin in these muscles had already been degraded.

MYHC is therefore the main target of many cachetic factors, but the mechanism of myosin loss varies and might be factor-specific. The authors also showed that loss of myosin leads to reduced complexes of myosin with its main binding partner, sarcomeric actin, but the downstream consequence of myosin loss needs to be investigated further.

6) References and links ORIGINAL RESEARCH PAPER Acharyya, S. et al. Cancer cachexia is regulated by selective targeting of skeletal muscle gene products. J. Clin. Invest. 114, 370-378 (2004)

FURTHER READING Tisdale, M. J. Cachexia in cancer patients. Nature Rev. Cancer 2, 862-872 (2002)

\section{THERAPEUTICS}

\section{Pinch hitter}

Although imatinib (Glivec) has revolutionized outcomes for people with chronic myelogenous leukaemia (CML), it is not perfect, as resistance is increasingly encountered. But now Charles Sawyers and colleagues have identified another compound - BMS-354825 - that seems to be even more potent than imatinib, and used together these drugs might offer the most powerful treatment yet for CML.

Imatinib inhibits the kinase activity of $\mathrm{BCR}-\mathrm{ABL}$, the translocation product that leads to CML. The ABL kinase switches back and forth between active and inactive forms, and imatinib seems to catch ABL in its inactive state and keep it that way. Drug sensitivity is lost when mutations lock $\mathrm{ABL}$ in an active state that imatinib can no longer access - so far, mutations at 17 different amino-acid positions within the BCR-ABL kinase domain have been found to confer clinical resistance. Sawyers and colleagues therefore examined other classes of ABL inhibitors for compounds that might block its kinase activity regardless of the active or inactive conformation of the enzyme.

They found that BMS-354825 is even more effective than imatinib at inhibiting BCR-ABL kinase activity in vitro. The drug also blocked the kinase activity of 14 out of 15 different clinically relevant, imatinib-resistant isoforms, as well the growth of the cells that expressed these proteins.

Furthermore, BMS-354825 markedly reduced tumour burden and prolonged survival of mice with either wild-type BCR-ABL-induced or imatinib-resistant disease. Importantly, BMS-354825 seems to be effective in human cells, as it inhibited the growth of bone-marrow progenitors isolated from patients with either imatinib-sensitive or imatinib-resistant CML.

If BMS-354825 proves safe and effective in clinical trials, it might be used in combination with imatinib to delay or prevent the emergence of drug-resistant disease. However, there is still at least one mutant form of BCR-ABL - T315I - that does not respond to either BMS-354825 in vitro or in vivo. Additional compounds will therefore need to be tested, as this mutation accounts for $15-20 \%$ of imatinib-resistant cases of CML.

Kristine Novak

\section{(2) References and links}

ORIGINAL RESEARCH PAPER Shah, N. P. et al. Overriding imatinib resistance with a novel ABL kinase inhibitor. Science 305, 399-401 (2004) WEB SITE

Charles Sawyer's lab: nsi.ucla.edu/faculty/sawyers_c.html

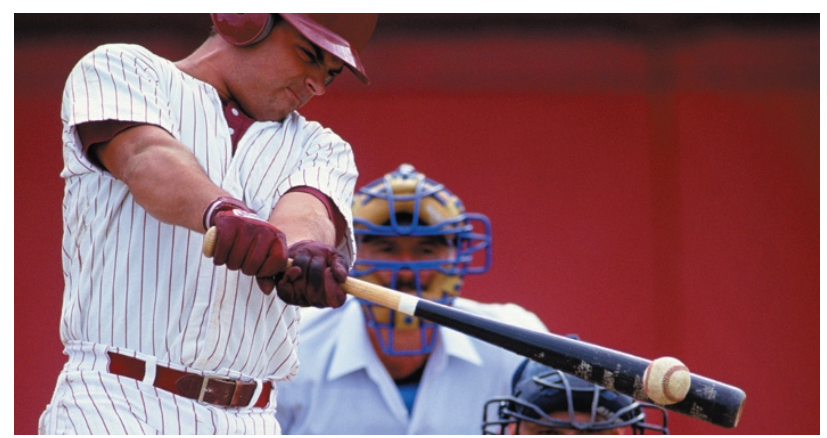

